Rev. Int. Contam. Ambie. 37, 443-453, 2021

https://doi.org/10.20937/RICA.53863

\title{
CARBONO DE LA BIOMASA MICROBIANA, RESPIRACIÓN BASAL Y CINÉTICA DEL CARBONO DE DOS SUELOS EROSIONADOS ENMENDADOS CON BIOSÓLIDOS
}

Soil biomass carbon, basal respiration and carbon kinetics of two eroded soils amended with biosolids

\author{
Nadia DE LA PORTILLA LÓPEZ, Rocío VACA PAULÍN, Pedro DEL ÁGUILA JUÁREZ, \\ Liliana SALINAS ALCÁNTARA, Gustavo YÁÑEZ OCAMPO y Jorge LUGO DE LA FUENTE*
}

Facultad de Ciencias, Universidad Autónoma del Estado de México, Instituto Literario 100, 50000 Toluca, Estado de México, México.

*Autor para correspondencia: jorgelug@gmail.com

(Recibido: enero de 2020; aceptado: octubre de 2020)

Palabras clave: medio edáfico, actividad respiratoria, materia orgánica, mineralización.

\section{RESUMEN}

El objetivo de esta investigación fue evaluar el efecto de la incorporación de tres dosis de biosólidos $(0,25$ y 40 t/ha) a suelos erosionados por extracción minera y erosión hídrica, sobre el carbono de la biomasa microbiana, la respiración basal y la cinética del carbono mineralizado a los 7, 30, 180 y 360 días después de dicha incorporación. Los resultados mostraron aumento de la respiración basal y el coeficiente metabólico en ambos suelos a los 7 y 360 días, respectivamente, después de la adición de 40 t/ha de biosólidos. En suelos erosionados por extracción minera el carbono de la biomasa microbiana fue mayor en el tratamiento control $\left(\mathrm{M}_{0}, 0 \mathrm{t} / \mathrm{ha}\right.$ de biosólido) después de 30 días de la incorporación, mientras que en el suelo con erosión hídrica el mayor contenido se presentó en $\mathrm{H}_{25}$ ( 25 t/ha de biosólido) durante los primeros 7 días. Finalmente, la adición de biosólidos favoreció la cinética de mineralización del carbono $(\mathrm{Cm})$ de manera que los suelos tratados presentaron valores más altos respecto al control.

Key words: edaphic medium, respiratory activity, organic matter, mineralization.

\begin{abstract}
The objective of this investigation was to evaluate the effect of the incorporation of three doses of biosolids $(0,25$ and $40 \mathrm{t} / \mathrm{ha}$ ) to soils eroded by mining extraction and water erosion, on the microbial biomass carbon, basal respiration and kinetics of mineralized carbon at 7, 30, 180 and 360 days after incorporating the biosolids. The results showed an increase in the basal respiration and the metabolic coefficient at 7 and 360 days in both soils, respectively, after the addition of $40 \mathrm{t} / \mathrm{ha}$ of biosolids. In the soils eroded by mining extraction, the microbial biomass carbon was higher in the control treatment $\left(\mathrm{M}_{0}, 0 \mathrm{t} / \mathrm{ha}\right.$ of biosolids) after 30 days of the incorporation, while for the soil with water erosion the highest content occured in $\mathrm{H}_{25}$ ( $25 \mathrm{t} /$ ha of biosolids) during the first seven days. Finally, the addition of biosolids favored the kinetics of carbon mineralization $(\mathrm{Cm})$ so that the treatments with biosolids presented higher values with respect to the control soil.
\end{abstract}




\section{INTRODUCCIÓN}

El suelo se modifica constantemente debido al efecto de las gotas de lluvia, la escorrentía, el viento, la gravedad y otros factores de formación, generando pérdida y transformación de energía y materia. De igual manera, las actividades antrópicas de minería, cambio de uso de suelo, urbanización, deforestación y prácticas agrícolas, entre otras, ocasionan cambios en las propiedades del suelo, provocando su erosión (Comino et al. 2017, Thomas et al. 2018).

La pérdida gradual de nutrientes y la reducción de materia orgánica $(\mathrm{MO})$ y carbono orgánico $(\mathrm{CO})$, así como la alteración de su estructura y fertilidad, son conocidos como erosión del suelo (Doni et al. 2017). Este fenómeno provoca la disminución de la actividad microbiana, afecta los ciclos biogeoquímicos y en consecuencia perjudica las propiedades bioquímicas del suelo (Sarapatka et al. 2018, Steinhoff-Knopp y Burkhard 2018, Yan et al. 2018), por lo que es necesario desarrollar estrategias para mitigarlo.

En la restauración de suelos, la incorporación de biosólidos como enmienda orgánica es una alternativa eficiente (Doni et al. 2017, Leila et al. 2017, Lombardi et al. 2017, Turunen et al. 2018). Los biosólidos se derivan del tratamiento de aguas residuales de origen doméstico o industrial $\mathrm{y}$, debido a su contenido de $\mathrm{N}, \mathrm{P}, \mathrm{MO}$ y micronutrientes, pueden utilizarse como enmiendas orgánicas que estimulan la actividad microbiana, mejorando las propiedades bioquímicas del suelo (Ociepa et al. 2017, Urbaniak et al. 2017, Arif et al. 2018, Mohamed et al. 2018). Diversos estudios señalan los efectos positivos de la incorporación de biosólidos sobre las propiedades bioquímicas de los suelos como resultado de su aporte de nutrimentos. Entre estos efectos destacan el aumento del carbono orgánico (COS), el nitrógeno, el carbono de la biomasa microbiana (CBM) y la respiración basal (RB), lo cual favorece la actividad microbiana y reactivación de los ciclos biogeoquímicos (Ros et al. 2003, Jiménez et al. 2007, Roig et al. 2012, Pardo et al. 2014, Arif et al. 2018, Siebieliec et al. 2018).

Las propiedades bioquímicas del suelo son indicadores de los cambios que se presentan en los procesos edáficos, debido a su sensibilidad ante los cambios o usos del suelo, su relación con la actividad microbiana y los procesos de mineralización del suelo. Las propiedades bioquímicas importantes del suelo son el carbono de la masa microbiana (CBM) y la respiración basal (RB) (Bera et al. 2016, Papp et al. 2018); esta última es el indicador ideal de la actividad microbiana y un parámetro sensible para monitorear la mineralización de la MO (García-Gil et al. 2004). Por otro lado, la cinética de la mineralización del carbono $(\mathrm{Cm})$ permite evaluar tanto el $\mathrm{C}$ potencialmente mineralizable y su tasa de mineralización como el C lábil que resulta de interés en la estimación de la capacidad de los suelos para suministrar carbono orgánico potencialmente mineralizable (Pedra et al. 2007, Martínez et al. 2008, Saviozzi et al. 2014). Por las razones anteriores, el objetivo del presente trabajo fue evaluar el efecto de la adición de biosólidos sobre el CBM, la RB y y la Cm en suelos con erosión hídrica y por extracción minera.

\section{MATERIALES Y MÉTODOS}

\section{Ubicación de las zonas de muestreo}

Se establecieron dos zonas de muestreo en el municipio Jiquipilco del Estado de México (México central) en función del tipo de erosión. La ubicación del suelo con grado fuerte de erosión hídrica $\left(19^{\circ} 31^{\text {, }}\right.$ $36^{\prime}$ " N, 99 41' 23 " W, con altitud de $2723 \mathrm{msnm}$ ) se tomó del trabajo realizado por Bolaños-González et al. (2016), en el cual se evaluaron las pérdidas de $\mathrm{CO}$ a nivel nacional a causa de erosión hídrica. La segunda zona fue en suelo erosionado por extracción minera, es decir, por extracción de arena $\left(19^{\circ} 32^{\prime} 35^{\prime \prime}\right.$ ' $\mathrm{N}, 99^{\circ} 43^{\prime} 54^{\prime}$ ' W, con altitud de $2746 \mathrm{~m}$ ). El clima de la zona se caracteriza por ser templado subhúmedo con lluvias en verano $\left.\left(\mathrm{C}_{\mathrm{w}} \mathrm{w}_{2}\right] \mathrm{w}\right)$, con temperatura media anual de $16.5^{\circ} \mathrm{C}$ y precipitación de $1128 \mathrm{~mm}$ (García 2004). El suelo para ambos sitios se clasifica como Phaeozem háplico (INEGI 1982).

\section{Toma de muestras de suelo y biosólido}

En agosto de 2017 se recolectaron en ambas zonas de estudio bloques de suelo (monolitos) de $28 \times 9$ $\times 16 \mathrm{~cm}$ (largo por ancho por alto, respectivamente) con ayuda de cincel, pala y martillo, los cuales fueron colocados de manera individual en recipientes de plástico identificados. Las muestras fueron transportadas al Laboratorio de Edafología y Ambiente de la Facultad de Ciencias de la UAEM para establecer el experimento.

En la misma fecha se recolectaron aproximadamente $40 \mathrm{~kg}$ de biosólidos (los cuales se depositaron en bolsas de polietileno) directamente del filtro prensa de la planta municipal de tratamiento de aguas residuales Toluca Norte de la empresa Operadora de Ecosistemas, ubicada en el municipio de Toluca, Estado de México, México.

\section{Descripción de los tratamientos}

Los tratamientos consistieron en adicionar tres dosis de biosólidos $(0,25$ y 40 t/ha) por cada tipo 
de erosión de suelo descrita en el cuadro I. Los biosólidos fueron adicionados en peso húmedo a los recipientes de plástico que contenían al suelo. La cantidad de biosólido varió, ya que el peso del suelo fue diferente en cada uno de los recipientes.

El experimento se realizó en condiciones de laboratorio con un diseño experimental de bloques completamente aleatorio, con cuatro repeticiones por tratamiento. En total constó de 24 muestras.

CUADRO I. DOSIS DE BIOSÓLIDOS UTILIZADAS PARA LA REALIZACIÓN DEL EXPERIMENTO.

\begin{tabular}{cl}
\hline Tratamiento & Descripción \\
\hline $\mathrm{H}_{0}$ & Suelo con erosión hídrica $+0 \mathrm{t} / \mathrm{ha}$ de biosólido \\
$\mathrm{H}_{25}$ & Suelo con erosión hídrica $+25 \mathrm{t} / \mathrm{ha}$ de biosólido \\
$\mathrm{H}_{40}$ & Suelo con erosión hídrica $+40 \mathrm{t} / \mathrm{ha}$ de biosólido \\
$\mathrm{M}_{0}$ & Suelo con erosión minera $+0 \mathrm{t} / \mathrm{ha}$ de biosólido \\
$\mathrm{M}_{25}$ & Suelo con erosión minera $+25 \mathrm{t} / \mathrm{ha}$ de biosólido \\
$\mathrm{M}_{40}$ & Suelo con erosión minera $+40 \mathrm{t} / \mathrm{ha}$ de biosólido \\
\hline
\end{tabular}

\section{Análisis de laboratorio}

Se seleccionó una muestra compuesta de $2 \mathrm{~kg}$ para cada zona de suelo erosionado antes del montaje del experimento, para realizar la caracterización física y química. Las muestras fueron secadas a temperatura ambiente, molidas y tamizadas a $2 \mathrm{~mm}$. La caracterización física y química de los suelos y el biosólido se realizó por separado, incluyendo la textura por el método de Bouyoucos (AS-09), la densidad aparente por el método de probeta, el $\mathrm{pH}$ medido en agua en relación 1:2.5 (método AS-02), la conductividad eléctrica por el método AS-18 y la MO por el método de Walkley y Black (método AS-07), todo ello con base en la NOM 021-2000-RECNAT (SEMARNAT 2002).

Los biosólidos fueron aplicados en peso húmedo en recipientes de plástico de $12 \times 35 \times 30 \mathrm{~cm}$ (largo por ancho por alto, respectivamente). Respecto a las determinaciones bioquímicas, se tomaron muestras de suelo a $0-20 \mathrm{~cm}$ de profundidad en cada uno de los monolitos con un sacabocados de PVC a los 7, 30, 180 y 360 días después de la aplicación del biosólido. Las muestras se colocaron en bolsas de polietileno transparentes previamente etiquetadas de las que se expulsó todo el aire. Finalmente se congelaron a $-24{ }^{\circ} \mathrm{C}$ para su posterior análisis.

Los análisis bioquímicos realizados fueron los siguientes: carbono de la biomasa microbiana por el método de fumigación-extracción (Vance et al. 1987); respiración basal del suelo mediante determinación de la emisión de $\mathrm{CO}_{2}$ por el método de Kassem y Nannipieri (1995); respiración a lo largo de 24 días y cinética de la mineralización del $\mathrm{C}$ a través de la metodología utilizada en Gil-Sotres et al. (1992).

El coeficiente metabólico es la cantidad de $\mathrm{C}-\mathrm{CO}_{2}$ emitido por unidad de biomasa y por hora $\left(q \mathrm{CO}_{2}\right)$, reportado para 24 días. Este concepto está basado en la teoría de sucesión de ecosistemas de Odum y se utiliza como un índice de desarrollo o perturbación del ecosistema (Anderson y Domsch, 1993).

\section{Análisis estadístico}

Se aplicó ANOVA para un diseño en bloques completamente aleatorio a los valores obtenidos de los análisis bioquímicos de los tratamientos. A los que presentaron diferencias estadísticas significativas $(\mathrm{p} \leq 0.05)$ se les aplicó la prueba de comparación múltiple de medias Tukey. Asimismo, se llevó a cabo una prueba de comparación de pendientes para detectar diferencias significativas en la cinética de mineralización del $\mathrm{C}$ en los diferentes tratamientos. Los análisis se realizaron empleando el paquete estadístico Statgrapichs Centurion 5.0 con un nivel de confianza del $95 \%$.

\section{RESULTADOS Y DISCUSIÓN}

\section{Caracterización física y química de los suelos y el biosólido}

La textura del suelo con erosión hídrica fue franco-arcillosa y para el de extracción minera fue franco-arcillo-arenosa, de acuerdo con la FAO. La densidad aparente obtenida fue de 1.02 y $1.13 \mathrm{~g} / \mathrm{ml}$ para $\mathrm{H}$ y $\mathrm{M}$, respectivamente. $\mathrm{El} \mathrm{pH}$ para ambos suelos se clasificó como moderadamente ácido $(\mathrm{H}$, 5.5; M, 6.4). Se presentaron efectos despreciables de salinidad en ambos suelos. La materia orgánica fue considerada de clase media en ambos suelos (NOM-021-RECNAT-2000 [SEMARNAT 2002]). El biosólido presentó un $\mathrm{pH}$ de 6.4 , una conductividad eléctrica de $3.70 \mathrm{ds} / \mathrm{cm}$ y un $45.09 \%$ de materia orgánica, siendo esta última ideal para el mejoramiento de las propiedades físicas, químicas y bioquímicas, lo que resulta benéfico para la restauración del suelo en términos de macro y micronutrientes (Samara et al. 2017, Skowrońska et al. 2020) (Cuadro II).

\section{Propiedades bioquímicas Respiración basal}

Los resultados mostraron para ambos suelos que a mayor dosis de biosólido adicionado, mayores valores de $\mathrm{RB}$, de manera que $\mathrm{H}_{40}$ y $\mathrm{M}_{40}$ presentaron 
CUADRO II. CARACTERIZACIÓN FÍSICA Y QUÍMICA DE SUELOS CON EROSIÓN HÍDRICA, MINERA Y BIOSÓLIDOS.

\begin{tabular}{lccc}
\hline Parámetro & Suelo con erosión hídrica & Suelo con erosión minera & Biosólido \\
\hline Clase textural & Franco-arcilloso & Franco-arcilloso-arenoso & ND \\
Arena (\%) & 43.7 & 55.4 & ND \\
Limo (\%) & 22.6 & 16.0 & ND \\
Arcilla $(\%)$ & 33.6 & 28.6 & ND \\
Densidad aparente $(\mathrm{g} / \mathrm{mL})$ & 1.02 & 1.13 & ND \\
pH* & $5.55 \pm 0.13$ & $6.40 \pm 0.07$ & $6.41 \pm 0.01$ \\
CE* $^{*}(\mathrm{ds} / \mathrm{cm})$ & $0.14 \pm 0.05$ & $0.08 \pm 0.01$ & $3.70 \pm 0.16$ \\
MO* $(\%)$ & $2.24 \pm 0.09$ & $2.69 \pm 0.63$ & $45.09 \pm 0.01$ \\
\hline
\end{tabular}

*Promedio \pm error estándar.

CE: conductividad eléctrica; MO: materia orgánica; ND: no determinado.

valores significativamente más altos y diferentes a los demás tratamientos $(\mathrm{p}<0.05)$. La tasa de respiración basal para ambos suelos erosionados se incrementó después de los primeros 5 días de incubación, mostrando aumento a lo largo del tiempo (Fig. 1). Respecto del tiempo, después de la adición de biosólidos el mayor aumento de RB se observó durante los primeros 7 días posteriores a la incorporación de la enmienda, a diferencia de los otros tiempos (30, 180 y 360 días después de incorporar los biosólidos). A los 7 días, el tratamiento $\mathrm{H}_{40}$ fue mayor en un 18 y $60 \%$ con relación a los tratamientos $\mathrm{H}_{25}$ y $\mathrm{H}_{0}$, respectivamente.

En el caso del suelo erosionado por extracción minera, el tratamiento $\mathrm{M}_{40}$ presentó un incremento del 37 y el $59 \%$ respecto a los tratamientos $\mathrm{M}_{25}$ y $\mathrm{M}_{0}$. De la misma manera, a los 360 días el suelo con erosión hídrica presentó el mayor incremento de $\mathrm{RB}$ en el tratamiento con la dosis más alta $\left(\mathrm{H}_{40}\right)$, superando a $\mathrm{H}_{25}$ y $\mathrm{H}_{0}$ en un 53 y $75 \%$, respectivamente. En el suelo con erosión por extracción minera sucedió lo mismo, ya que el tratamiento con la dosis más alta $\left(\mathrm{M}_{40}\right)$ presentó incrementos del 30 y el $47 \%$ con relación a $\mathrm{M}_{25}$ y $\mathrm{M}_{0}$. Los incrementos de $\mathrm{RB}$ relacionados con la dosis alta se han asociado con la gran cantidad de MO presente en el biosólido, la cual restituye la $\mathrm{MO}$ del suelo con un valor medio de aproximadamente $60 \%$ peso seco, pero con un intervalo que puede fluctuar entre el 35 y el $80 \%$ (Urbaniak et al. 2017). Este comportamiento también se puede atribuir a la entrada de $\mathrm{C}$ fácilmente disponible hacia los microorganismos y a la nueva biomasa que está creciendo en el biosólido (Andrés et al. 2011). Dicha contribución energética estimula la actividad heterótrofa del suelo, lo cual se ve reflejado en aumento de la actividad respiratoria presente en los microorganismos del suelo (Peña et al. 2015).
Con relación al periodo de tiempo en que se presentó la mayor tasa de respiración (medición al día 7), éste puede deberse a que los biosólidos promueven en corto tiempo un efecto positivo en la actividad respiratoria, ya que la incorporación de sustancias lábiles estimula la actividad microbiana del suelo y acelera la tasa de mineralización, lo cual se ve reflejado prontamente en mayor actividad respiratoria (Mardomingo et al. 2013). Esto podría resultar benéfico para reestablecer la calidad y productividad de los suelos erosionados debido a que los biosólidos aceleran la acumulación de MO y estimulan la actividad microbiana (Mikha et al. 2014).

\section{Cinética de mineralización del C $(\mathrm{Cm})$}

La figura 2 representa la cinética de mineralización del $\mathrm{C}(\mathrm{Cm})$ durante los 24 días de incubación utilizados para la actividad respiratoria. Los resultados muestran que la tendencia de las gráficas en ambos suelos erosionados se ajusta a un modelo de cinética de primer orden $\left(\mathrm{R}^{2}>0.848\right)$ (Gil-Sotres 1992). Por otro lado, el análisis de varianza mostró diferencias no significativas en la velocidad de mineralización entre los tratamientos ( $\mathrm{p}>0.05)$; sin embargo, se observó ligera tendencia de descenso de la $\mathrm{Cm}$ al aumentar la dosis de biosólido, de tal forma que $\mathrm{H}_{40}$ y $\mathrm{M}_{40}$ presentaron los valores más bajos de $\mathrm{Cm}$ (Cuadro III). Lo anterior se puede atribuir a las fuentes de $\mathrm{C}$ lábil proporcionadas por los biosólidos, las cuales utilizan los microorganismos del suelo como sustratos fácilmente degradables. Las formas de $\mathrm{C}$ lábil (hidratos de carbono o compuestos orgánicos de bajo peso molecular) que se proceden del C orgánico son esenciales para la actividad biológica, ya que los organismos (mayoritariamente heterótrofos) las utilizan como recurso energético. Los componentes lábiles de la fracción de carbono orgánico del suelo 


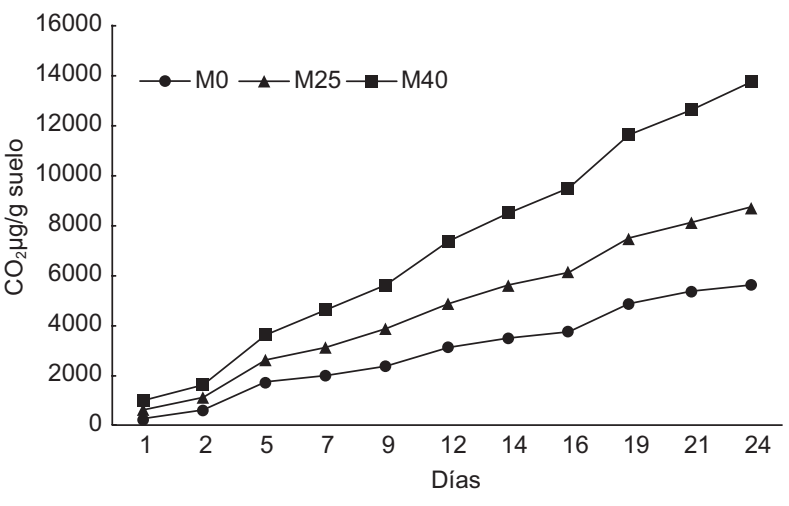

7 días

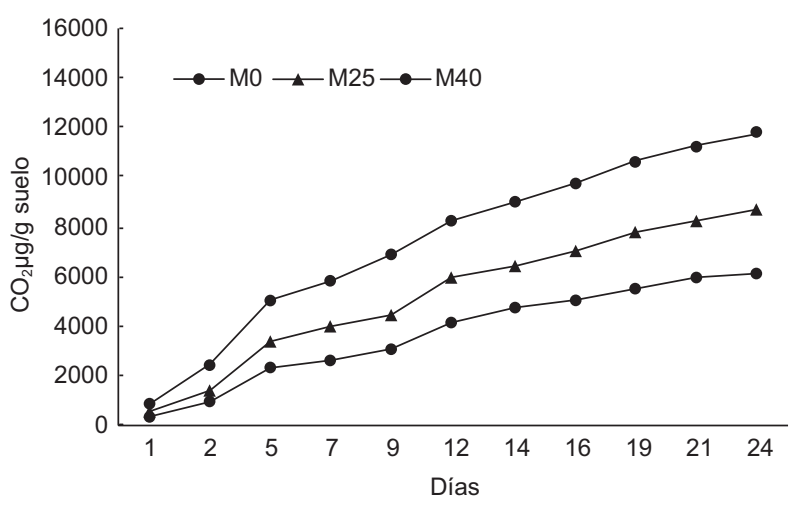

30 días
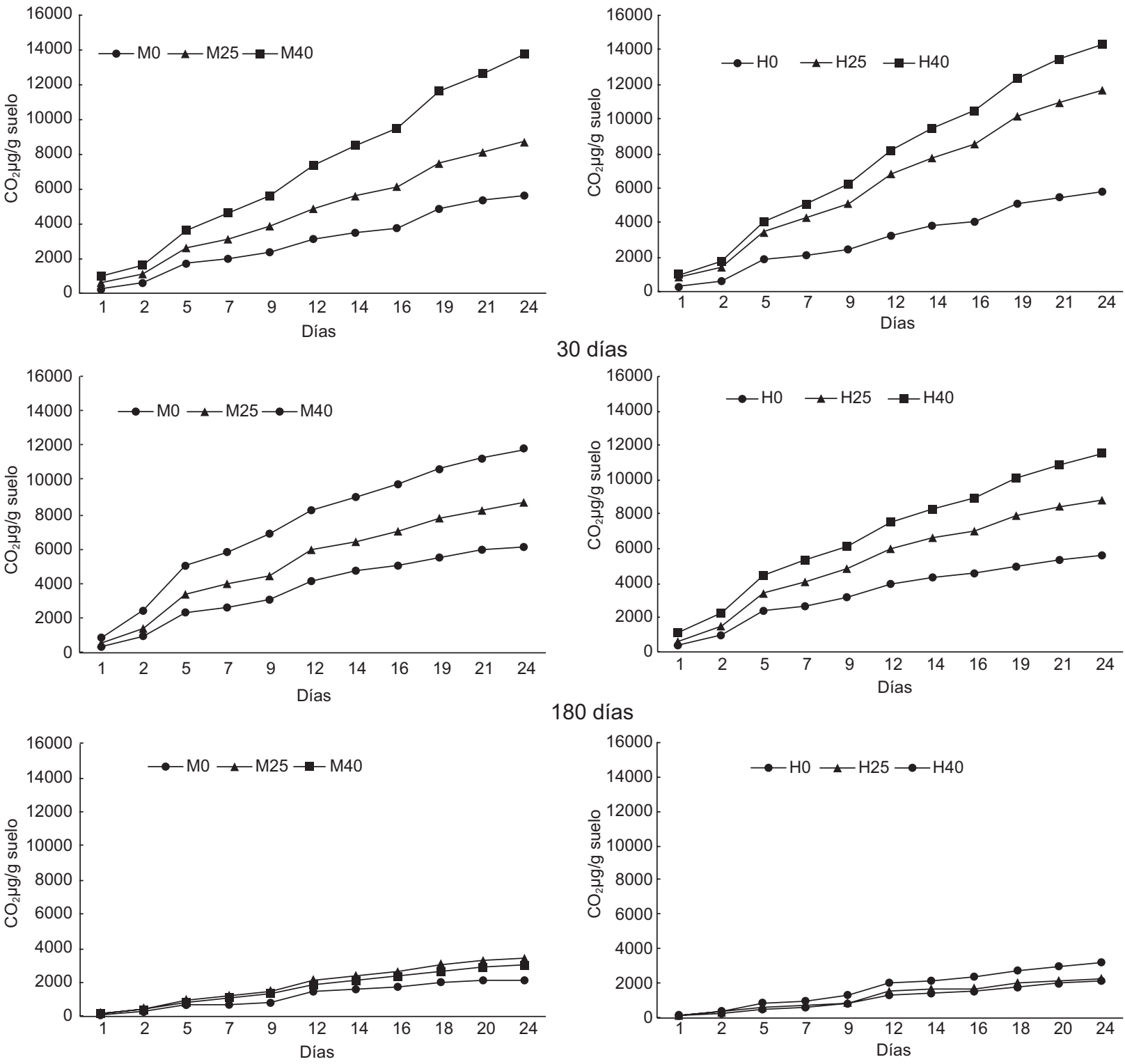

180 días
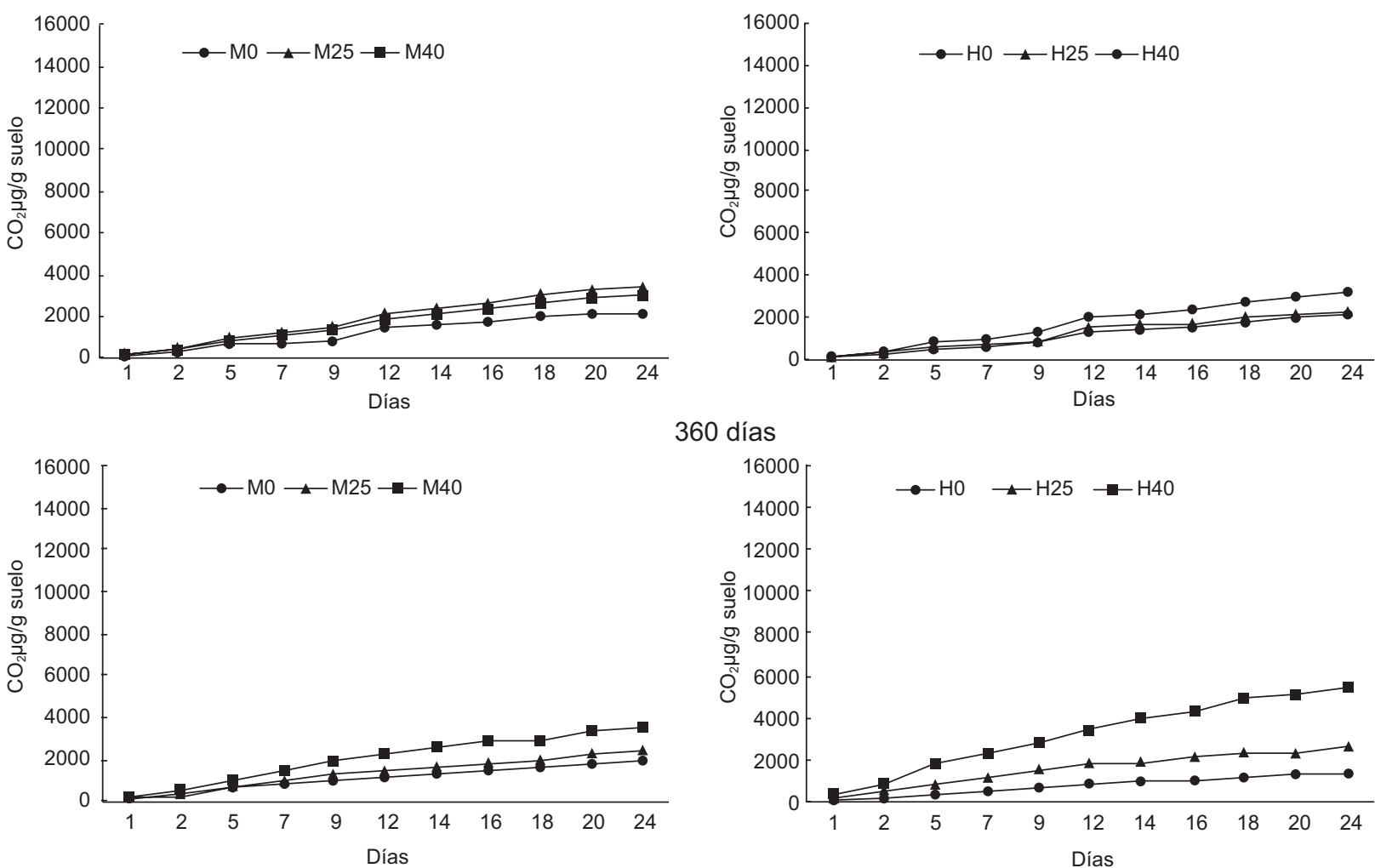

360 días

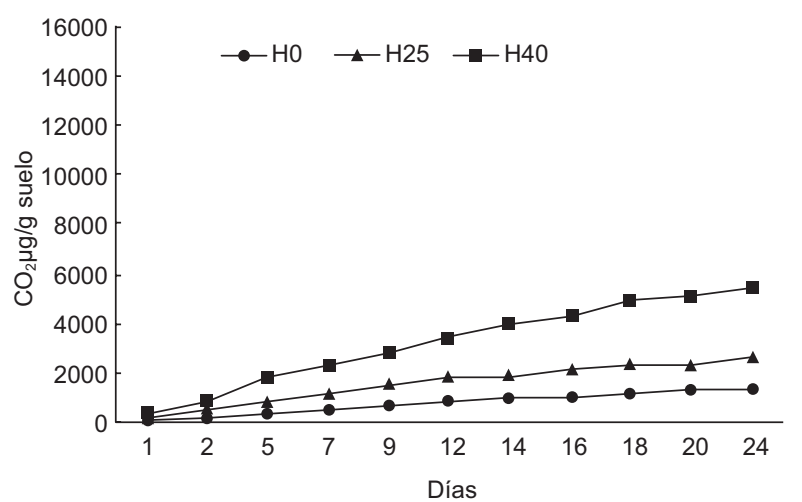

Fig. 1. Respiración basal de suelos con erosión enmendados con biosólidos a los 7, 30, 180 y 360 días después de la aplicación de los tratamientos. $\mathrm{M}_{0}, \mathrm{M}_{25}$ y $\mathrm{M}_{40}$ : suelos con erosión por extracción minera enmendados con 0,25 y 40 t/ha de biosólido, respectivamente; $\mathrm{H}_{0}, \mathrm{H}_{25}$ y $\mathrm{H}_{40}$ : suelos con erosión hídrica enmendados con 0,25 y 40 t/ha de biosólido, respectivamente. 
7 días
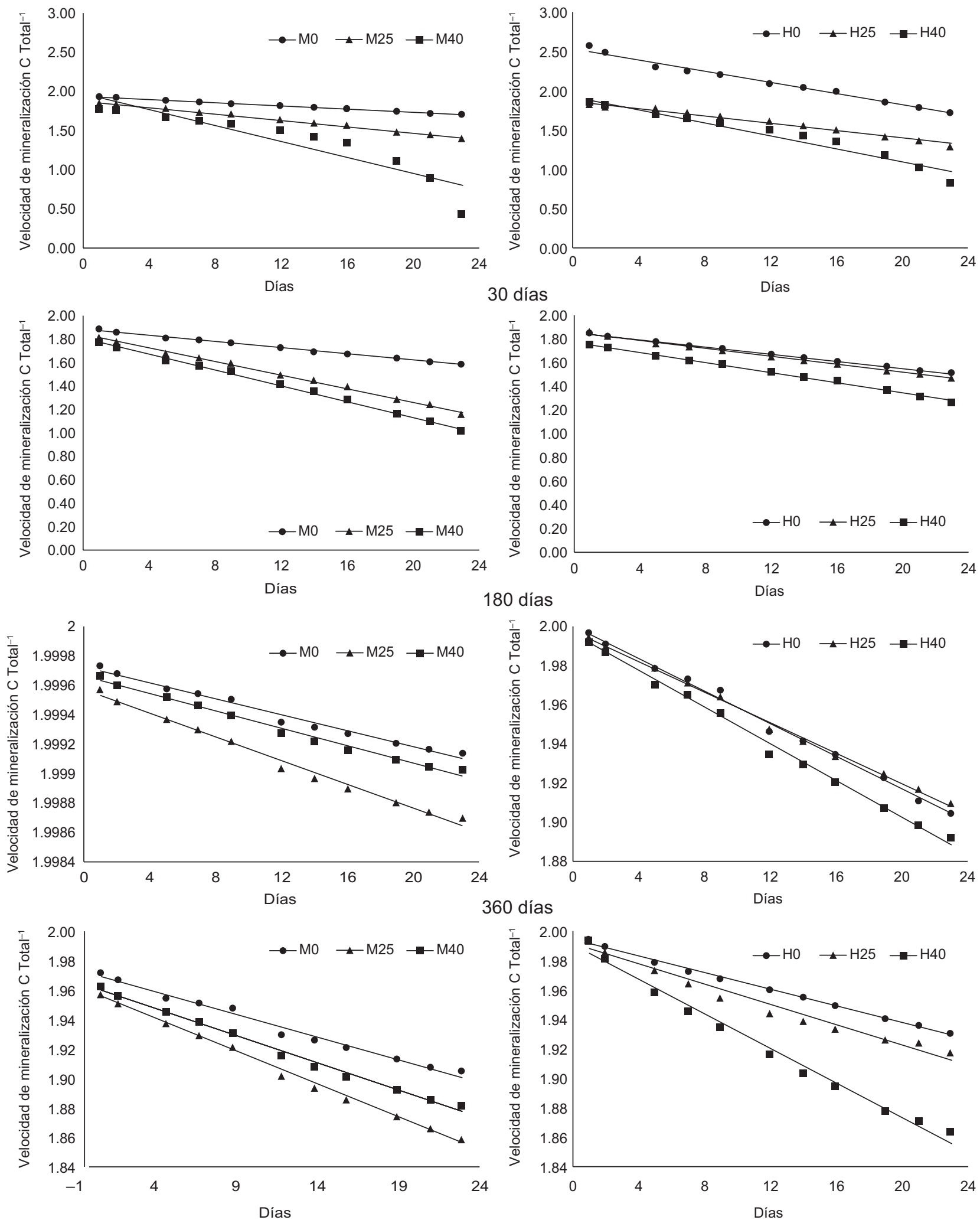

Fig. 2. Cinética de mineralización del carbono $(\mathrm{Cm})$ en suelos con erosión enmendados con biosólidos a los 7, 30, 180 y 360 días después de la aplicación de los tratamientos. $\mathrm{M}_{0}, \mathrm{M}_{25} \mathrm{y} \mathrm{M}_{40}$ : suelos con erosión por extracción minera enmendados con $0,25 \mathrm{y}$ 40 t/ha de biosólido, respectivamente; $\mathrm{H}_{0}, \mathrm{H}_{25}$ y $\mathrm{H}_{40}$ : suelos con erosión hídrica enmendados con 0,25 y 40 t/ha de biosólido, respectivamente. 
CUADRO III. AJUSTE DE LAS RECTAS DE DOS SUELOS EROSIONADOS ENMENDADOS CON BIOSÓLIDOS A DIFERENTES DOSIS Y TIEMPOS.

\begin{tabular}{|c|c|c|c|}
\hline & $\mathrm{R}^{2}$ & $\mathrm{y}$ & $\mathrm{p}$ \\
\hline \multicolumn{4}{|c|}{7 días } \\
\hline $\mathrm{H}_{0}$ & 0.987 & $y=2.531-3.548 \times 10^{-2}$ & $\mathrm{p}<0.05$ \\
\hline $\mathrm{H}_{25}$ & 0.986 & $\mathrm{y}=1.863-2.313 \times 10^{-2}$ & $\mathrm{p}<0.05$ \\
\hline $\mathrm{H}_{40}$ & 0.938 & $\mathrm{y}=1.920-4.127 \times 10^{-2}$ & $\mathrm{p}<0.05$ \\
\hline $\mathrm{M}_{0}$ & 0.986 & $\mathrm{y}=1.936-1.010 \times 10^{-2}$ & $\mathrm{p}<0.05$ \\
\hline $\mathrm{M}_{25}$ & 0.983 & $\mathrm{y}=1.872-2.036 \times 10^{-2}$ & $\mathrm{p}<0.05$ \\
\hline $\mathrm{M}_{40}$ & 0.848 & $\mathrm{y}=1.971-5.091 \times 10^{-2}$ & $\mathrm{p}<0.05$ \\
\hline \multicolumn{4}{|c|}{30 días } \\
\hline $\mathrm{H}_{0}$ & 0.988 & $\mathrm{y}=1.852-1.518 \times 10^{-2}$ & $\mathrm{p}<0.05$ \\
\hline $\mathrm{H}_{25}$ & 0.996 & $y=1.855-1.674 \times 10^{-2}$ & $\mathrm{p}<0.05$ \\
\hline $\mathrm{H}_{40}$ & 0.995 & $y=1.770-2.125 \times 10^{-2}$ & $\mathrm{p}<0.05$ \\
\hline $\mathrm{M}_{0}$ & 0.992 & $\mathrm{y}=1.886-1.311 \times 10^{-2}$ & $\mathrm{p}<0.05$ \\
\hline $\mathrm{M}_{25}$ & 0.995 & $\mathrm{y}=1.842-2.912 \times 10^{-2}$ & $\mathrm{p}<0.05$ \\
\hline $\mathrm{M}_{40}$ & 0.995 & $\mathrm{y}=1.807-3.388 \times 10^{-2}$ & $\mathrm{p}<0.05$ \\
\hline \multicolumn{4}{|c|}{180 días } \\
\hline $\mathrm{H}_{0}$ & 0.995 & $\mathrm{y}=2.00-4.157 \times 10^{-3}$ & $\mathrm{p}<0.05$ \\
\hline $\mathrm{H}_{25}$ & 0.993 & $\mathrm{y}=1.998-3.894 \times 10^{-3}$ & $\mathrm{p}<0.05$ \\
\hline $\mathrm{H}_{40}$ & 0.994 & $\mathrm{y}=1.997-4.691 \times 10^{-3}$ & $\mathrm{p}<0.05$ \\
\hline $\mathrm{M}_{0}$ & 0.981 & $y=2.000-2.707 \times 10^{-5}$ & $\mathrm{p}<0.05$ \\
\hline $\mathrm{M}_{25}$ & 0.990 & $\mathrm{y}=2.000-4.027 \times 10^{-5}$ & $\mathrm{p}<0.05$ \\
\hline $\mathrm{M}_{40}$ & 0.988 & $\mathrm{y}=2.000-2.953 \times 10^{-5}$ & $\mathrm{p}<0.05$ \\
\hline \multicolumn{4}{|c|}{360 días } \\
\hline $\mathrm{H}_{0}$ & 0.992 & $\mathrm{y}=1.994-2.823 \times 10^{-3}$ & $\mathrm{p}<0.05$ \\
\hline $\mathrm{H}_{25}$ & 0.968 & $\mathrm{y}=1.991-3.446 \times 10^{-3}$ & $\mathrm{p}<0.05$ \\
\hline $\mathrm{H}_{40}$ & 0.987 & $y=1.991-5.874 \times 10^{-3}$ & $\mathrm{p}<0.05$ \\
\hline $\mathrm{M}_{0}$ & 0.984 & $\mathrm{y}=1.972-3.125 \times 10^{-3}$ & $\mathrm{p}<0.05$ \\
\hline $\mathrm{M}_{25}$ & 0.994 & $\mathrm{y}=1.961-4.531 \times 10^{-3}$ & $\mathrm{p}<0.05$ \\
\hline $\mathrm{M}_{40}$ & 0.992 & $y=1.964-3.748 \times 10^{-3}$ & $\mathrm{p}<0.05$ \\
\hline
\end{tabular}

$\mathrm{H}_{0}, \mathrm{H}_{25}$ y $\mathrm{H}_{40}$ : suelos con erosión hídrica enmendados con 0,25 y 40 t/ha de biosólido, respectivamente; $\mathrm{M}_{0}, \mathrm{M}_{25}$ y $\mathrm{M}_{40}$ : suelos con erosión por extracción minera enmendados con 0,25 y 40 t/ha de biosólido, respectivamente; $\mathrm{p}$ : probabilidad.

son los principales responsables de la rápida tasa inicial de mineralización del C; por tanto, influyen en gran medida en los ciclos de nutrientes del suelo (García-Gil et al. 2004, Martínez et al. 2008, Mohanty et al. 2013).

\section{Carbono de la biomasa microbiana}

El contenido de CBM en el suelo con erosión hídrica se incrementó después de la aplicación de biosólidos, principalmente con $\mathrm{H}_{25}$, que a los 7 días produjo valores altos y significativamente diferentes $(\mathrm{p}<$ 0.05 ) respecto a los demás tratamientos (Cuadro IV). Lo anterior se atribuye a que los biosólidos son fuente importante de MO y C fácilmente biodegradable, disponible para los microorganismos presentes en el suelo y para la nueva biomasa microbiana que se encuentra en crecimiento en el biosólido. Dichos organismos tienen la capacidad de generar mayor actividad microbiana en los procesos de ciclos de nutrientes y en la descomposición, conversión y suministro de MO (Selivanovskaya et al. 2000, Andrés et al. 2011, Mondal et al. 2015, Bargali et al. 2018).

La incorporación de biosólidos no incrementó significativamente $(p>0.05)$ el contenido de CBM en el suelo con erosión por extracción minera, de manera que el valor más alto se presentó a los 7 y 30 días en $\mathrm{M}_{0}$, seguido de $\mathrm{M}_{25}$ y $\mathrm{M}_{40}$. Este comportamiento se observó hasta los 180 días de medición (Cuadro V). Lo anterior se puede atribuir a que la actividad minera tiene efectos negativos sobre los nutrientes del suelo. Esto se ve reflejado en disminución del $\mathrm{C}$ y de la actividad de los microorganismos, la cual es inhibida a pesar de la aplicación de biosólidos (que introduce gran cantidad de microorganismos) debido a que las poblaciones naturales del suelo intentan permanecer homeostáticas (Sastre et al. 1996, Ruiz et al. 2018, Guo et al. 2019).

\section{$q \mathrm{CO}_{2}$}

En el suelo con erosión hídrica, el coeficiente metabólico $\left(q \mathrm{CO}_{2}\right)$ aumentó en los tratamientos con $25 \mathrm{o}$ $40 \mathrm{t} / \mathrm{ha}$ en todos los tiempos, excepto a los 7 días de haberse incorporado el biosólido. En el caso del suelo erosionado por extracción minera, $q \mathrm{CO}_{2}$ aumento en los tratamientos con 25 y 40 t/ha de biosólido en todos los tiempos, a excepción de 360 días después de la aplicación del biosólido (Cuadros IV y V). Al igual que la respiración del suelo, $q \mathrm{CO}_{2}$ es un indicador de la actividad microbiana y de las modificaciones ocurridas en el suelo, por lo que es útil para evaluar los efectos de la aplicación de biosólidos (Pavan et al. 2005). El aumento de $q \mathrm{CO}_{2}$ con la dosis alta de biosólido se puede atribuir al estrés microbiano causado por la mayor cantidad de energía gastada por los microorganismos para su mantenimiento, como resultado del aumento en la disponibilidad de una fuente de $\mathrm{C}$ lábil por unidad de biomasa (García-Gil et al. 2004, Pascual et al. 2007). Dicho estrés se asocia con un menor contenido de biomasa y la capacidad de ejercer una mayor eficiencia metabólica ante la presencia de la MO contenida en los biosólidos.

\section{CONCLUSIONES}

La adición de 40 t/ha de biosólido incrementó la respiración basal, la cinética de mineralización del 

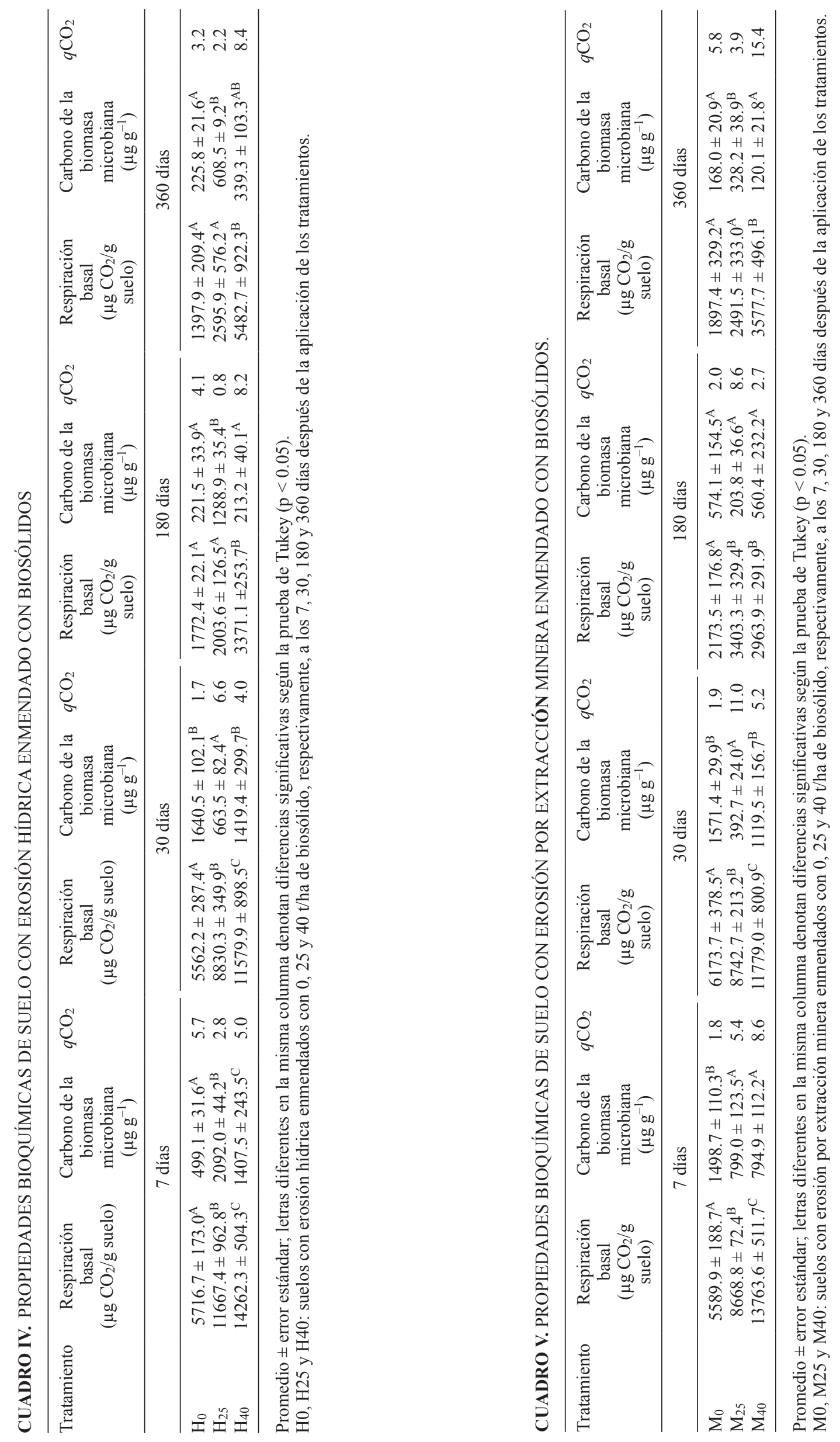
carbono y el coeficiente metabólico, tanto en el suelo con erosión hídrica como en el suelo con erosión por extracción minera.

El carbono de la biomasa microbiana presentó los valores más altos en el suelo control en el caso de la erosión por extracción minera, mientras que en el suelo con erosión hídrica los valores más altos se alcanzaron en el tratamiento con adición de $25 \mathrm{t} /$ ha de biosólido.

La cinética de mineralización del carbono disminuyó en los tratamientos con 40 t/ha en ambos suelos con relación a los otros tratamientos.

\section{REFERENCIAS}

Anderson T.H. y Domsch K.H. (1993). The metabolic quotient for $\mathrm{CO}_{2}\left(q \mathrm{CO}_{2}\right)$ as a specific activity parameter to assess the effects of environmental conditions, such as $\mathrm{pH}$, on the microbial biomass of forest soils. Soil Biol. Biochem. 25 (3), 393-395. https://doi. org/10.1016/0038-0717(93)90140-7

Andrés P., Mateos E., Tarrasón D., Cabrera C. y Figuerola B. (2011). Effects of digested, composted, and thermally dried sewage sludge on soil microbiota and mesofauna. Appl. Soil Ecol. 48 (2), 236-242. https:// doi.org/10.1016/j.apsoil.2011.03.001

Arif M.S., Riaz M., Shahzad S.M., Yasmeen T., Ashraf M., Siddique M., Mubarik M.S., Bragazza L. y Buttler A. (2018). Fresh and composted industrial sludge restore soil functions in surface soil of degraded agricultural land. Sci. Total Environ. 619-620, 517-527. https://doi. org/10.1016/j.scitotenv.2017.11.143

Bargali K., Manral V., Padalia K., Bargali S.S. y Upadhyay V.P. (2018). Effect of vegetation type and season on microbial biomass carbon in Central Himalayan forest soils, India. Catena 171, 125-135. https://doi. org/10.1016/j.catena.2018.07.001

Bera T., Collins H.P., Alva A.K., Purakayastha T.J. y Patra A.K. (2016). Biochar and manure effluent effects on soil biochemical properties under corn production. Appl. Soil Ecol. 107, 360-367. https://doi. org/10.1016/j.apsoil.2016.07.011

Bolaños-González M.A., Paz-Pellat F., Cruz-Gaistardo C.O., Argumedo-Espinoza J.A., Romero-Benítez V.M. y de la Cruz-Cabrera J.C. (2016). Mapa de erosión de los suelos de México y posibles implicaciones en el almacenamiento de carbono orgánico del suelo. Terra Latinoamericana 34 (3), 271-288.

Comino J.R., Senciales J.M., Ramos M.C., MartínezCasasnovas J.A., Lasanta T., Brevik E.C., Ries J.B. y Ruiz-Sinoga J.D. (2017). Understanding soil erosion processes in Mediterranean sloping vineyards (Montes de Málaga, Spain). Geoderma 296, 47-59. https://doi. org/10.1016/j.geoderma.2017.02.021

Doni S., Macci C., Longo V., Souid A., Garcia C. y Masciandaro G. (2017). Innovative system for biochemical monitoring of degraded soils restoration. Catena 152, 173-181. https://doi.org/10.1016/j.catena.2017.01.016

García E. (2004). Modificaciones al sistema de clasificación climática de Koppen. 5a ed. Serie Libros. Instituto de Geografía, Universidad Nacional Autónoma de México. 91 pp.

García-Gil J.C., Plaza C., Senesi N., Brunetti G. y Polo A. (2004). Effects of sewage sludge amendment on humic acids and microbiological properties of a semiarid Mediterranean soil. Biol. Fertil. Soils 39, 320-328. https://doi.org/10.1007/s00374-003-0709-z

Gil-Sotres F., Trasar-Cepeda M.C., Ciardi C., Ceccanti B. y Leirós M.C. (1992). Biochemical characterization of biological activity in very young mine soils. Biol. Fertil. Soils 13, 25-30. https://doi.org/10.1007/BF00337233

Guo Z., Han J., Li J., Xu Y. y Wang X. (2019). Effects of long-term fertilization on soil organic carbon mineralization and microbial community structure. PLoS ONE 14 (1), 1-16. https://doi.org/10.1371/journal. pone. 0211163

INEGI (1982). Carta edafológica Ixtlahuaca E-14-A-27. Escala 1:50 000. Instituto Nacional de Estadística y Geografía, México.

Jiménez J., Ortiz O., Tarrasón D., Ginovart M. y Bonmatí M. (2007). Effect of differently post-treated dewatered sewage sludge on $\beta$-glucosidase activity, microbial biomass carbon, basal respiration and carbohydrates contents of soils from limestone quarries. Biol. Fertil. Soils 44, 393-398. https://doi.org/10.1007/s00374007-0220-z

Kassem A. y Nannipieri P. (1995). Methods in applied soil microbiology and biochemistry. Academic Press, EUA, 608 pp.

Leila S., Mhamed M., Hermann H., Mykola K., Oliver W., Christin M., Onyshchenkoc E. y Bouchenafa N. (2017). Fertilization value of municipal sewage sludge for Eucalyptus camaldulensis plants. Biotechnol. Rep. 13, 8-12 https://doi.org/10.1016/j.btre.2016.12.001

Lombardi L., Nocita C., Bettazzi E., Fibbi D y Carnevale E. (2017). Environmental comparison of alternative treatments for sewage sludge: An Italian case study. Waste Manage. 69, 365-376. https://doi.org/10.1016/j. wasman.2017.08.040

Mardomingo J.I., Soler-Rovira P., Casermeiro M.A., de la Cruz M.T y Polo A. (2013). Seasonal changes in microbial activity in a semiarid soil after application of a high dose of different organic amendments. Geoderma 206, 40-48. https://doi.org/10.1016/j.geoderma.2013.04.025 
Martínez H.E., Fuentes E.J.P. y Acevedo H.E. (2008). Carbono orgánico y propiedades del suelo. Rev. Cienc. Suelo Nutr. 8 (1), 68-96. https://doi.org/10.4067/ S0718-27912008000100006

Mikha M., Benjamin J., Stahlman P. y Geier, P. (2014). Remediation/restoration of degraded soil: I. Impact on soil chemical properties. Soil Till. Res. 106 (1), 252260. https://doi.org/10.2134/agronj2013.0278

Mohamed B., Olivier G., François G., Laurence A.L., Bourgeade P., Badr A.S. y Lotfi A. (2018). Sewage sludge as a soil amendment in a Larix decidua plantation: Effects on tree growth and floristic diversity. Sci. Total Environ. 621, 291-301. https://doi.org/10.1016/j. scitotenv.2017.11.283

Mohanty S., Nayak A., Kumar A., Tripathi R., Shahid M., Bhattacharyya P., Raja R. y Panda B. (2013). Carbon and nitrogen mineralization kinetics in soil of rice system under long term application of chemical fertilizers and farmyard manure. Eur. J. Soil Biol. 58, 113-121. https://doi.org/10.1016/j. ejsobi.2013.07.004

Mondal S., Singh R., Patra A. y Dwivedi B. (2015). Changes in soil quality in response to short-term application of municipal sewage sludge in a typic haplustept under cowpea-wheat cropping system. Environ. Nanotechnol. Monit. Manag. 4, 37-41. https://doi.org/10.1016/j. enmm.2014.12.001

Ociepa E., Mrowiec M. y Lach J. (2017). Influence of fertilisation with sewage sludge-derived preparation on selected soil properties and prairie cordgrass yield. Environ. Res. 156, 775-780. https://doi.org/10.1016/j. envres.2017.05.003

Papp R., Marinari S., Moscatelli M.C., van der Heijden M.G.A., Wittwer R., Campigliac E., Radicettic E., Mancinellic R., Fradgleyd N., Pearcee B., Bergkvistf G. y Finckhg M.R. (2018). Short-term changes in soil biochemical properties as affected by subsidiary crop cultivation in four European pedo-climatic zones. Soil Till. Res. 180, 126-136. https://doi.org/10.1016/j. still.2018.02.019

Pardo T., Clemente R., Alvarenga P. y Bernal M. (2014). Efficiency of soil organic and inorganic amendments on the remediation of a contaminated mine soil: II. Biological and ecotoxicological evaluation. Chemosphere 107, 101108. https://doi.org/10.1016/j.chemosphere.2014.03.017

Pascual I., Antolín C., García C., Polo A. y SánchezDíaz M. (2007). Effect of water deficit on microbial characteristics in soil amended with sewage sludge or inorganic fertilizer under laboratory conditions. Bioresour. Technol. 98 (1), 29-37. https://doi.org/10.1016/j. biortech.2005.11.026

Pavan S., Bettiol W. y Clementi C. (2005). Effect of sewage sludge on microbial biomass, basal respira- tion, metabolic quotient and soil enzymatic activity. Appl. Soil Ecol. 30, 65-77. https://doi.org/10.1016/j. apsoil.2004.03.008

Pedra F., Polo A., Ribeiro A. y Domingues H. (2007). Effects of municipal solid waste compost and sewage sludge on mineralization of soil organic matter. Soil Biol. Biochem. 39, (1375-1382). https://doi. org/10.1016/j.soilbio.2006.12.014

Peña A., Mingorance M. y Rossini-Oliva S. (2015). Soil quality improvement by the establishment of a vegetative cover in a mine soil added with composted municipal sewage sludge. J. Geochem. Explor. 157, 178-183. https://doi.org/10.1016/j.gexplo.2015.06.014

Roig N., Sierra J., Martí E., Nadal M., Schuhmacher M. y Domingo J.L. (2012). Long-term amendment of Spanish soils with sewage sludge: Effects on soil functioning. Agric. Ecosyst. Environ. 158, 41-48. https://doi. org/10.1016/j.agee.2012.05.016

Ros M., Hernández M.T. y García C. (2003). Bioremediation of soil degraded by sewage sludge: Effects on soil properties and erosion losses. Waste Manage. 31 (6), 741-747. https://doi.org/10.1007/ s00267-002-2839-8

Ruiz C., Caro-Moreno D., Jiménez-Cantizano F., Macías F. y Pérez-López R. (2018). Assessing the quality of potentially reclaimed mine soils: Environmental implications for the construction of a nearby water reservoir. Chemosphere 216, 19-30. https://doi.org/10.1016/j. chemosphere.2018.09.018

Samara S., Matsi T. y Balidakis A. (2017). Soil application of sewage sludge stabilized with steelmaking slag and its effect on soil properties and wheat growth. Waste Manage. 68, 378-387. https://doi.org/10.1016/j.wasman.2017.06.016

Saviozzi A., Vanni G. y Cardelli R. (2014). Carbon mineralization kinetics in soils under urban environment. Appl. Soil Ecol. 73, 64-69. https://doi.org/10.1016/j. apsoil.2013.08.007

Sarapatka B., Cap L. y Bila P. (2018). The varying effect of water erosion on chemical and biochemical soil properties in different parts of Chernozem slopes. Geoderma 314, 20-26. https://doi.org/10.1016/j.geoderma.2017.10.037

Sastre I., Vicente M. y Lobo M. (1996). Influence of the application of sewage sludges on soil microbial activity. Bioresour. Technol. 57 (1): 19-23. https://doi. org/10.1016/0960-8524(96)00035-1

Selivanovskaya S., Latypova V., Kiyamova S. y Alimova F. (2000). Use of microbial parameters to assess treatment methods of municipal sewage sludge applied to grey forest soils of Tatarstan. Agric. Ecosyst. Environ. 86, 145-153 https://doi.org/10.1016/S01678809(00)00281-4 
SEMARNAT (2002). Norma Oficial Mexicana NOM021-RECNAT-2000. Que establece las especificaciones de fertilidad, salinidad y clasificación de suelos, estudio, muestreo y análisis. Secretaría de Medio Ambiente y Recursos Naturales. Diario Oficial de la Federación, 31 de diciembre.

Siebielec G., Siebielec S. y Lipski D. (2018). Long-term impact of sewage sludge, digestate and mineral fertilizers on plant yield and soil biological activity. J. Clean. Prod. 187, 372-379. https://doi.org/10.1016/j. jclepro.2018.03.245

Skowrońska M., Bielińska E., Szymański K., Futa B., Antonkiewicz J. y Kołodziej B. (2020). An integrated assessment of the long-term impact of municipal sewage sludge on the chemical and biological properties of soil. Catena 189, 104484 https://doi.org/10.1016/j. catena.2020.104484

Steinhoff-Knopp B. y Burkhard B. (2018). Soil erosion by water in Northern Germany: Long term monitoring results from Lower Saxony. Catena 165, 299-309. https://doi.org/10.1016/j.catena.2018.02.017
Thomas J., Joseph S. y Thrivikramj K.P. (2018). Assessment of soil erosion in a tropical mountain river basin of the southern Western Ghats, India using RUSLE and GIS. Geosci. Front. 9 (3), 893-906. https://doi. org/10.1016/j.gsf.2017.05.011

Turunen V., Sorvari J. y Mikola A. (2018). A decision support tool for selecting the optimal sewage sludge treatment. Chemosphere 193, 521-529. https://doi. org/10.1016/j.chemosphere.2017.11.052

Urbaniak M., Wyrwicka A., Tołoczko W., Serwecińska L. y Zieliński M. (2017). The effect of sewage sludge application on soil properties and willow (Salix sp.) cultivation. Sci. Total Environ. 586, 66-75. https://doi. org/10.1016/j.scitotenv.2017.02.012

Vance E.D., Brookes P.C. y Jenkinson D.S. (1987). An extraction method for measuring soil microbial biomass C. Soil Biol. Biochem. 19, 703-707. https://doi. org/10.1016/0038-0717(87)90052-6

Yan R., Zhang X., Yan S. y Chen H. (2018). Estimating soil erosion response to land use/cover change in a catchment of the Loess Plateau, China. Int. Soil Water Conserv. Res. 6, 13-22. https://doi.org/10.1016/j. iswcr.2017.12.002 